\title{
Idealización y transmisión psíquica en la adolescencia: interpretación de un evento de violencia en Chile
}

\author{
Daniel Jofré* \\ Universidad Austral de Chile, Instituto de Psicología. Puerto Montt, Región de los Lagos, Chile
}

\begin{abstract}
Resumen: El propósito de este artículo es examinar el asesinato de un joven homosexual, perpetrado en Chile en 2012, y articular este evento de violencia a los imaginarios de la sociedad chilena contemporánea y a los procesos de idealización y de transmisión psíquica. Con este objetivo, se desarrolla una interpretación teórica que vincula elaboraciones psicoanalíticas sobre el rol del superyó cultural y de la idealización durante el pasaje adolescente, y el entrecruzamiento de la dialéctica étnico-política y la dialéctica ético-moral en la Teoría de la Mediación. El vínculo propuesto resalta el poderío de los ideales sociales de dominio y de rechazo del otro sobre los procesos de apropiación subjetiva de la adolescencia. Así, se propone un acercamiento a este evento de violencia que busca entretejer los procesos psíquicos e interpsíquicos de la adolescencia y los procesos de subjetivación constitutivos de la experiencia social de los adolescentes y jóvenes del Chile actual.
\end{abstract}

Palabras clave: pasaje adolescente, persona, violencia, idealización y transmisión.

\section{Introducción}

El propósito de este artículo es examinar el asesinato de un joven homosexual, por parte de un grupo de jóvenes chilenos, e intentar articular este evento de violencia a los imaginarios de la sociedad chilena contemporánea y a los procesos de idealización y de transmisión psíquica.

Nuestra argumentación posee la siguiente organización. En la primera parte, se presenta el crimen y se analizan las connotaciones que adquiere el asesinato, perpetrado durante 2012, del joven homosexual D. $Z$. dentro de la sociedad chilena. En este punto, se describen las reacciones sociales frente a este hecho de violencia, pero también se abre la pregunta respecto de su relación con los imaginarios sociales del Chile contemporáneo. En la segunda parte, intentaremos comprender las principales dimensiones en juego en este caso. Con este propósito, a partir de datos conocidos públicamente, se propone una interpretación según la cual, a la base de esta manifestación excesiva de violencia, se encuentra la detención o el impedimento de los procesos que orientan el posicionamiento del adolescente en lo social, obturando los procesos de elaboración y de transmisión psíquica.

Por consecuencia, se propone un acercamiento, que integra los procesos psíquicos e interpsíquicos presentes en la adolescencia y los efectos de individualización sobre la juventud chilena, que se conforman a partir de los discursos sociales del Chile actual. En este sentido, se intentará dar cuenta de los vínculos que sería admisible reconocer, entre procesos psíquicos como la sublimación o la idealización, y formas de subjetivación,

* Dirección para correspondencia: jofre.daniel@gmail.com que constitutivas de la experiencia social de los jóvenes chilenos, conforman un horizonte problemático, en cuanto proponen y legitiman la segregación, la violencia y el rechazo a la alteridad.

\section{Primera parte}

\section{El crimen y sus repercusiones}

El 2 de marzo de 2012, el joven homosexual Daniel Zamudio es atacado y torturado durante algunas horas por un grupo de jóvenes. El ataque tuvo lugar en un parque en el centro de la ciudad de Santiago, capital de Chile. El 27 de marzo el joven muere después de algunas semanas pasadas en vano en el hospital para intentar curar los traumatismos producidos por el ataque. ${ }^{1} \mathrm{El}$ asesinato del joven homosexual tuvo un fuerte impacto sobre la población a causa de la naturaleza de las

1 El propósito del presente artículo consiste en abordar posibles articulaciones entre los procesos sociohistóricos que configuran la sociedad chilena contemporánea y este evento de violencia, razón por la cual, no se profundizará en la historia personal de sus participantes, limitándonos a entregar sólo algunos elementos necesarios para la visualización del caso. Por tanto, no se busca indagar en los móviles explícitos o en las motivaciones psicológicas que entregan los involucrados sobre el evento que se les imputa, en cuanto, de hecho, respecto de este punto, las declaraciones de los jóvenes son zigzagueantes: tres de los imputados niegan su participación en los hechos durante el proceso judicial, mientras que uno de ellos reconoce su participación, pero declara que esta obedecería al miedo insuperable a sufrir igual trato que la víctima. Con todo, recomendamos al lector interesado en indagar en mayores precisiones sobre la individualización de los imputados en este caso, así como, sobre otros antecedentes relevantes para ponderar este evento de violencia, los siguientes documentos de acceso público: Individualización de audiencia de lectura de sentencia: https://bit.ly/2Mdkpld, Daniel Zamudio, hiciste historia: https://bit.ly/2yxx7WA, Estándares conceptuales y cargas procesales en el litigio antidiscriminación. Análisis crítico de la jurisprudencia sobre Ley Zamudio entre 2012 y 2015: https:// bit.ly/2K8iiuV 
heridas: grave daño en el cráneo, ruptura de miembros, quemaduras de cigarrillos y marcas sobre el estómago del joven hombre; como también, por el móvil explícito del crimen, la orientación sexual del joven asesinado. Todo lo anterior, contribuyó a generar un amplio debate social sobre las minorías sexuales y los crímenes de odio en la sociedad chilena.

Desde el punto de vista jurídico y policial, esta muerte interroga la necesidad de dar una significación a una serie de acciones perpetradas, aparentemente, sin justificación. Así, para el Ministerio Público de Chile (Cuarto Tribunal de Juicio Oral en lo Penal de Santiago, 2013), se trataba de una violencia que aspiraba, principalmente, a la humillación y a la tortura del joven homosexual, de manera que los actos son presentados desde una perspectiva que pone el acento en el completo control del otro y en el desprecio absoluto de la persona de D. Z. De hecho, las alegaciones del Ministerio Público de Chile durante la apertura del juicio se esfuerzan por subrayar la capacidad de los jóvenes involucrados en el crimen de producir un daño completamente desproporcionado a la víctima, con el objeto de demostrar el carácter ignominioso de sus actos:

se trata de una historia de horror, padecimiento infinito de la víctima, lesiones inhumanas, sesiones de torturas, desprecio por la vida e indolencia ante el sufrimiento de una víctima que no podía oponer resistencia. En cuanto a la agravante de ignominia, hubo una afectación a la dignidad de la víctima, que se acreditará a través de esvásticas de su cuerpo, quemaduras de cigarrillos, esta repetición de golpes dada en forma conjunta por todos los acusados, a ninguno le importó lo mal que estaba la víctima. (Cuarto Tribunal de Juicio Oral en lo Penal de Santiago, 2013, pp. 10-11)

Para la opinión pública, de forma incontestable, los jóvenes implicados en este asesinato cumplen con todos los atributos de la barbarie. El calificativo escogido para dar cuenta de esta condición es el de neonazis (calificativo con el cual, por otra parte, los jóvenes asesinos gustaban autodefinirse). De esta forma, el crimen de D.Z. adquiere una connotación que expresa la necesidad social de encapsular a los jóvenes asesinos como una parte ajena y extraña al conjunto de la sociedad. Cierto o no, este esfuerzo por cristalizar en un grupo de jóvenes una imagen estereotipada del odio y la discriminación poseyó una gran efectividad dentro del medio social chileno.

La cristalización de la violencia en la imagen de estos cuatro jóvenes genera, sin embargo, algunas interrogantes. Cuestionamientos, que buscan comprender el vínculo entre este evento de violencia y los imaginarios de la sociedad chilena; respecto del otro, la diferencia y la violencia.

\section{Algunas indicaciones sobre la sociedad chilena actual}

Los procesos colectivos que fundan los imaginarios sociales del Chile contemporáneo se inscriben dentro de la trama sociohistórica, cuyo principal impacto en la sociedad ha consistido en la adopción de un modelo social, jurídico y económico que insta a una continua privatización de los vínculos sociales y a una profunda individualización de las relaciones entre las personas y lo social.

Estudios recientes en el campo de la psicopatología han intentado comprender la importancia que adquiere este proceso histórico y social para la significación de la propia subjetividad, dando lugar a una hipótesis, que sitúa el impacto de los procesos de individualización de la sociedad chilena en la interioridad de los sujetos, mediante la identificación de éstos a ideales de emprendimiento, bienestar, rendimiento y competencia (Aceituno, Miranda, \& Jiménez, 2012).

Por otra parte, estudios sociológicos, que se abocan a conocer el ideario nacional construido durante la post dictadura y su impacto sobre la población, insisten en la creación de un imaginario social que se impone al individuo mediante narraciones de autonomía y una creciente segregación social (Mayol, Azócar, \& Azócar, 2012; Moulian, 2002; Salazar \& Valderrama, 2000). Este imaginario colectivo sostendría, a juicios de estos análisis, la necesidad de distanciarse y diferenciarse del otro, presente entre los ciudadanos del Chile contemporáneo.

Canales (2007) observa en este mismo sentido que las dificultades de integración o de inclusión de grupos mayoritarios dentro de la población chilena son la expresión de una profunda rigidez y estratificación social, que consolida un patrón de relaciones sociales de carácter discriminador. Resulta importante subrayar que, de acuerdo con este estudio, la motivación subyacente a estos actos de discriminación radicaría en la necesidad de dominar y disminuir al otro, con el objeto de sostener un ideal de homogeneidad y jerarquía social. Estas apreciaciones son compartidas por una parte importante de las investigaciones históricas y antropológicas. Estas investigaciones, observan que, en la sociedad chilena, la tendencia histórica al rechazo de la alteridad se expresa en la relación de los individuos respecto de sí mismos y de la sociedad, y encuentra una ilustración particularmente clara, dentro de hechos cotidianos y manifestaciones más o menos espontaneas de violencia, frente a grupos como las mujeres, los inmigrantes o las minorías sexuales (Aravena Reyes \& Baeza, 2013; Duarte, 2007; Salazar, 1999).

Se podría concluir esta breve revisión de estas hipótesis sobre la violencia en la sociedad chilena indicando: (1) la profundidad de los procesos de individualización y privatización, constitutivos de los actuales modos de interacción social; (2) la interrelación entre los patrones de conductas, los modos de vida y las cosmovisiones 
históricas de la sociedad chilena; y (3) la construcción de una imagen del otro al menos problemática, que remite a la diferenciación y a la violencia.

\section{Segunda parte}

\section{Elaboración de una posición de análisis}

Las elaboraciones que desarrollamos a partir de este caso no buscan establecer una relación cronológica o psicogenética, a través de la cual sea posible suponer el fracaso de un proceso evolutivo, de índole psicológico, para los jóvenes involucrados en el asesinato de D.Z. Al contrario, se busca generar una reflexión que, considerando los desafíos propios del trabajo adolescente, permita subrayar la importancia de los procesos de historización y de elaboración transgeneracional al momento de examinar la articulación entre los procesos psíquicos de la adolescencia y la inscripción del joven dentro de la sociedad.

El acento de nuestro análisis se concentra en la importancia de elaborar durante el pasaje adolescente las disposiciones narrativas de una sociedad que introduce la violencia, la discriminación y el dominio, como formas válidas de interacción social. En este sentido, este caso posee un gran valor interpretativo, no solamente por la particularidad de las vivencias de precariedad y desprotección experimentadas por los jóvenes involucrados, sino también porque permite representar las tensiones, individuales y colectivas, producidas por imaginarios sociales constituyentes de formas violentas de subjetivación, en donde el otro figura como un enemigo potencial o un antagonista a quien descalificar o humillar.

Considerando lo anterior, nuestra interpretación se organiza a partir de las siguientes referencias. Por una parte, a nivel de las instancias psíquicas concernidas en el proceso de elaboración adolescente, sería a nuestro juicio posible situar estas disposiciones narrativas en las exigencias e imperativos del superyó cultural respecto del sujeto. Por otra parte, nuestra lectura se apoya en los desarrollos sobre el entrecruzamiento de la dialéctica étnico-política y la dialéctica ético-moral, presentes en la Teoría de la Mediación.

La noción de superyó cultural, mencionada en la obra de Freud (1929/1978) en El malestar en la cultura, reconoce la importancia de esta instancia psíquica en los procesos de transmisión transgeneracional, como también respecto del vínculo entre el estado de las prescripciones colectivas que organizan un determinado entorno social y el superyó del individuo. La referencia de Freud (1929/1978) a la noción de superyó cultural admite, dentro de las exigencias conscientes e inconscientes ejercidas por el superyó, una fuente colindante con la historia personal y familiar, que imprime, a través del trabajo cultural, las características de una época y una fuente que reactualiza la conflictividad entre la cultura y el mundo pulsional.
De esta forma, el superyó de la cultura expresará las exigencias éticas que reglamentan las prácticas y relaciones sociales, constituyendo la fuente desde la cual se nutren los ideales de una sociedad. Así, a través del vínculo conjeturado por Freud (1921/1978) en Psicología de las masas y análisis del yo, entre las disposiciones colectivas y los mecanismos psíquicos subyacentes a la identificación del sujeto a la masa, es posible diferenciar entre el impulso a la idealización del sujeto - que, al alero de la ambivalencia y el narcisismo infantil, introduce de modo constante nuevas exigencias para el sujeto-y los procesos de sublimación. Como también, es posible subrayar el impacto que estos mecanismos y procesos psíquicos tienen en las formaciones éticas que reglamentan las acciones individuales y colectivas.

Ahora bien, dentro de los autores que desarrollan la noción de Pasaje adolescente, se apuesta por comprender que, en el vínculo entre el sujeto y el superyó, se expresan las tensiones entre el orden discursivo de una época o cultura y el trabajo de transmisión y de posicionamiento subjetivo que enfrenta el sujeto adolescente. En primer lugar, J. J. Rassial (1996) examina el concepto de superyó cultural haciendo referencia - no sin cierta ambigüedad - a la noción de superyó colectivo, en alusión a la articulación entre el sujeto y el Otro. Así, a través de esta noción, este autor referirá tanto a una autoridad exterior, arcaica, que permite la entrada del sujeto al vínculo social, como al discurso del Amo, en cuanto dispositivo fundador del lazo social. En segundo lugar, O. Douville (2005) notará la correspondencia entre las formulaciones estructuralistas de Lacan, respecto de la escritura del sujeto de lo inconsciente, y la participación del sujeto dentro del sistema del lenguaje y los intercambios sociales, legales, económicos, míticos o religiosos, que conforman su universo cultural. De suerte que, dentro de la escritura del sujeto de lo inconsciente, la instancia psíquica del superyó será, para este autor, el espacio en donde el sujeto experimentará en forma invertida el discurso del Otro. Serge Lesourd (2006), basado en estas indicaciones, referirá mediante la noción de superyó cultural, una producción representativa del conjunto de exigencias éticas que reglamentan las relaciones sociales, para una época determinada y que integraría tanto los pequeños relatos como los grandes relatos, de orden mítico o colectivo. Por consiguiente, la figura del superyó cultural viene a representar dentro de estos análisis un conjunto de ideas, imaginarios e imperativos que, ocupando un lugar en la interioridad psíquica del sujeto, serían también demostrativos del lugar de exterioridad que ocupan las narraciones sociales para el sujeto. Se pone de relieve, en este sentido, la lógica que liga al sujeto a las disposiciones socioculturales y que, articulada bajo la forma del superyó, organiza los posicionamientos y elaboraciones del sujeto adolescente dentro de los discursos que propician formas específicas de vínculo social. 
El enfoque dialéctico propuesto por la Teoría de la Medicación de Jean Gagnepain (1993) busca poner de relieve que el comportamiento cultural del hombre - a nivel del lenguaje, de los útiles, de la ley social y de la norma - se encuentra mediado por una serie de tiempos lógicos - condición natural, análisis formal e investidura positiva- que permiten al individuo establecer una distancia respecto de su relación al mundo y poner en marcha un proceso reflexivo que, no obstante, introduce constantemente la negatividad como una fracción irresoluble por estas mediaciones culturales.

Dentro de este modelo, la dialéctica étnicopolítica o de la persona, tiene por objeto dilucidar las mediaciones que permiten comprender la relación del sujeto a los vínculos y discursos instituyentes de su horizonte sociocultural. Al interior de esta dialéctica, por lo tanto, se observan las modalidades según las cuales se expresa la articulación entre la singularidad de la experiencia y el orden colectivo, mediante un continuo proceso de apropiación subjetiva del orden instituyente. Cabe resaltar que, para la Teoría de la Mediación, este proceso que permite el reposicionamiento del adolescente dentro de las lógicas sociales admite remarcar el reconocimiento de la historicidad y arbitrariedad de los vínculos sociales, y el surgimiento de la responsabilidad del individuo frente a su posicionamiento generacional e intergeneracional. Por otra parte, la Teoría de la Mediación reconoce en la dialéctica ético-moral o de la norma, la capacidad del individuo de analizar su comportamiento, estableciendo las restricciones y habilitaciones que, durante la infancia y más tarde en la adolescencia y la vida adulta, son admisibles dentro del contexto en donde se desarrolla. El esquema propuesto por Jean Gagnepain (1993) permite, en consecuencia, diferenciar el ámbito de relación del individuo con la Ley social y la capacidad del individuo de analizar su propio deseo y reglamentar su comportamiento. Será, pues, en la interacción entre los procesos que permiten la relativización de las exigencias sociales y la capacidad humana de auto limitar su comportamiento que emergerá la potencialidad de someter a análisis los imperativos e ideales, representativos del horizonte cultural, familiar o grupal, en el que habita el individuo.

Así, desde la Teoría de la Mediación (Gagnepain, 1993; Quentel, 2011, 2015), a nivel de los procesos dialécticos presentes en la adolescencia, se resalta la importancia del trabajo psíquico e intersubjetivo al que insta el entrecruzamiento de la dialéctica de la persona y la norma, que permite: (1) comprender el carácter relativo de la organización social y de las diferentes narrativas sociales (pero también grupales), e (2) impulsa al adolescente a analizar las propias actuaciones, pensamientos y discursos, tomando en consideración la necesidad de otorgarles sentido y legitimidad.

Es posible poner de relieve, por lo tanto, que la construcción propuesta pretende indagar sobre las posibles interrelaciones que, en el tiempo de la adolescencia, sería posible reconocer dentro del proceso de posicionamiento subjetivo frente al superyó cultural y a la capacidad del individuo de delimitar la influencia de los códigos sociales y grupales en su comportamiento. Ciertamente, cabe señalar a este respecto que, a nivel epistemológico, resulta necesario reconocer la especificidad y particularidad de los horizontes conceptuales que movilizan la noción de pasaje adolescente y los desarrollos de la Teoría de la Mediación. De hecho, los modelos de interpretación psicoanalíticos sobre la adolescencia tienen como ámbito de análisis primordial las simbolizaciones humanas que permiten la inscripción del sujeto a nivel del deseo y la falta durante el trabajo de la adolescencia. Por su parte, respecto de estas cuestiones, el modelo de análisis presente en la obra de Jean Gagnepain busca comprender las mediaciones individuales e intersubjetivas que desarrolla el adolescente respecto tanto del orden social como de su propio comportamiento. El vínculo propuesto entre ambos modelos, en consecuencia, resalta el poderío de los ideales sociales - que reniegan del horizonte simbólico e histórico de los vínculos humanos - sobre los procesos de subjetivación y apropiación subjetiva de la adolescencia, aspecto cuya importancia es remarcada por ambos modelos teóricos.

De este modo, se propone un acercamiento a este evento de violencia capaz de entretejer, los procesos psíquicos e interpsíquicos de la adolescencia y los procesos de subjetivación constitutivos de la experiencia social de los adolescentes y jóvenes del Chile actual.

Existe, sin embargo, otro aspecto interviniente en la comprensión de esta propensión a la violencia presente en la sociedad chilena contemporánea, el cual se vincula a la idealización de la violencia como fenómeno y mecanismo psicológico. En efecto, en el testimonio de estos jóvenes, en su forma de torturar y humillar a D.Z., se encuentra algo que es posible calificar como una fascinación por el poder que se puede ejercer sobre el otro. ${ }^{2}$ Una suerte de virilización de la violencia que se

2 El lector puede ponderar esta apreciación sobre las acciones de los involucrados en este evento de violencia a partir de los alegatos del Ministerio Público en la lectura de sentencia por este delito. En este sentido, remitimos a los siguientes párrafos de esta lectura, en donde consideramos que se transmite la fuerza desmedida ejercida sobre la víctima y la escaza sensibilidad frente a su sufrimiento.

Se han omitido los nombres de los imputados con el objeto de resguardar su privacidad. Cabe señalar, no obstante, que la lectura de sentencia completa se encuentra en la siguiente referencia Web: http://www. movilh.cl/documentacion/Sentencia-Daniel-Zamudio-Vera.pdf

"Daniel se recuesta en el suelo y se quedó dormido; A dice que lo va a despertar y le pega con el talón en la sien y en la nuca, luego B. Ellos dos comienzan a pegarle continuamente, saltaban en su cabeza y nariz. A él le dijeron que no se podía ir, se quedó sentado en un árbol que estaba en la misma bajada, luego que lo golpearon, se sentaron, siguieron fumando. Posteriormente B se levantó y orinó en la boca y pecho de Zamudio, luego A hizo lo mismo, también le orinó encima, después le siguen pegando; A le rompe una botella de ron Mitjans en la cabeza de Zamudio y B toma el gollete y le hace tres esvásticas, le hace dos en el estómago y una el pecho y le da dos "punzazos" en el lado izquierdo del cuerpo" (Cuarto Tribunal de Juicio Oral en lo Penal de Santiago, 2013, p. 63).

"A y B le pegan nuevamente a Daniel, zamarrean brutalmente su cabeza tomándola de las orejas y golpeándola, rebotaba en el suelo, ve que también le pega $\mathrm{C}$, pero de una manera más suave, como obligado, luego A rompe 
representa en la elección de una estética o estilo juvenil, en donde la fuerza y la agresividad son insignias que representan a estos jóvenes ante otros. Sería posible, en este sentido, pensar que la violencia opera también como una figura, una imagen o una narrativa que impide la crítica autónoma respecto de los ideales del grupo.

Obsérvese a este respecto que la idealización de la violencia, comprendida en tanto que proceso psíquico tendiente a atribuir al objeto idealizado (narrativas históricas y sociales sobre la violencia, el otro, la jerarquía social, etc.) la totalidad de las perfecciones, impide la apertura a procesos sublimatorios capaces de reestablecer una distancia respecto de los requerimientos del superyó e interrumpe la creación de un tiempo y un espacio para el trabajo psíquico de deconstrucción de los sistemas referenciales del sujeto (Gutton, 2002; Lesourd, 2004; Mijolla-Mellor, 2012).

\section{Un esfuerzo interpretativo: la relación entre marginación social y la idealización de figuras imaginarias de todo poder y dominio}

Dentro de los antecedentes recogidos y analizados para comprender la actuación de los jóvenes presentes en el asesinato, se constata un fuerte impulso a la ejecución de actos de violencia. Como si, desde el poderío de las referencias identitarias en las cuales estos jóvenes escogen acogerse, se abriera finalmente un espiral de referencias imaginarias, a partir de las cuales se reconocieran a sí mismos bajo una óptica patriarcal, autoritaria y fálica. Nótese, no obstante, que la ambigüedad de estas referencias descansaría en la necesidad de sobrellevar, a través de ellas, la fragilidad de experiencias transidas por pérdidas, abandonos y violencia ${ }^{3}$.

De este modo, sería posible inferir: emerge como recurso psíquico e intersubjetivo, la idealización de la violencia. Idealización de una violencia confusa, que se podría suponer, se introduce como un elemento suplementario dentro de escenas imaginarias de todo poder y control, a través de las cuales estos jóvenes transforman sus propias vivencias de precariedad y desprotección. Sin embargo, también se podría sostener que, mediante estos procesos de idealización, los jóvenes obturan el trabajo de elaboración de sus historias y fantasmas y el proceso de apropiación e historización de las narrativas grupales y sociales. Por consiguiente, en lugar del trabajo

otra botella en la cabeza de Zamudio y le dice a B que aprenda a hacer los cortes y le hace otras dos esvásticas en la espalda, luego B le hace otro corte en el cuello; A le dice que ese corte está mejor. Después A y B toman un "camote" y se lo tiran en la cabeza y el cuerpo constantemente" (Cuarto Tribunal de Juicio Oral en lo Penal de Santiago, 2013, p. 64).

3 Nos permitimos referir a este respecto el trabajo de R. Fluxá (2014), que, dentro de los marcos de una investigación periodística, da cuenta de la importante precarización e intensas vivencias de desafiliación experimentadas por los jóvenes involucrados en el asesinato de D. Z. El autor resalta a este respecto que la totalidad de los jóvenes involucrados presentan dentro de sus relaciones familiares fuertes conflictividades, que remiten, dependiendo del caso, a la falta de reconocimiento de la paternidad, disoluciones familiares o la violencia doméstica. de elaboración identitaria propio de la adolescencia, nos encontraríamos con un exceso de heteronomía, una complacencia excesiva frente a imperativos superyoicos que impulsan a la repetición de actos de violencia y al desprecio por otros y su diferencia.

Obsérvese en este punto algunas precisiones del marco interpretativo propuesto para una adecuada ponderación de la argumentación. La dialéctica de la persona, en tanto que expresión de la racionalidad humana presente en el vínculo del individuo al orden colectivo, designa un proceso permanente $\mathrm{y}$, de algún modo, atemporal. Sin embargo, como lo observa J. L. Brackelaire (2009), esta dialéctica designa también la apertura a la construcción de una temporalidad y de una espacialidad propia de los procesos individuales y sociales, que tiene como resultado el despliegue de un trabajo permanente de apropiación y de reapropiación. Desde esta óptica, este autor propone comprender, por suspensión de la persona, una forma de alteración de esta disposición a la historización, a causa principalmente del profundo énfasis en la singularización del orden socio discursivo contemporáneo, que impide una verdadera apertura a la alteridad. Esta suspensión, por lo tanto, no es ni individual ni colectiva, sino que compromete la estructuración implícita y la organización de estas relaciones. De parte de las elaboraciones psicoanalíticas, cabe resaltar los señalamientos que realiza Olivier Douville (2001) respecto de la neutralización de los procesos de transmisión entre las generaciones. En efecto, este autor utiliza la noción de melancolización del lazo social para poner de relieve los impedimentos subjetivos presentes en sujetos que dentro de su historia vivida o heredada han sido confrontados a figuras de todo poder, que impiden la asignación y la traducción de la pertenencia, como también la construcción de alianzas simbólicas de reciprocidad e intercambio con la alteridad. Estos impedimentos, por consecuencia, serán demostrativos del vínculo imaginario del sujeto a la historia y a los discursos colectivos: modo de relación con la propia historia y con la historia colectiva, habilitado para forjar un impasse en el trabajo subjetivo de historización durante el momento adolescente del fantasma, en los procesos de transmisión transgeneracional y de posicionamiento en el vínculo social.

Se trata por lo tanto de subrayar, dentro de los procesos dialécticos que permiten la subjetivación adolescente, el impedimento de reencontrar la alteridad y de transmitir la propia historia y la historia colectiva. En este sentido, se propone comprender este impedimento de los procesos de subjetivación y de apropiación subjetiva como producto del entrecruzamiento de discursos sociales ultra individualistas y de los traumatismos psicológicos y sociales, causados por continuas vivencias de desprotección y abuso, tanto a nivel de la historia personal, como colectiva. De suerte que, es posible sostener que el impasse subjetivo que atraviesan estos jóvenes sería la expresión de una suerte de encapsulamiento imaginario del trabajo 
de la adolescencia, el cual impide la disolución de los anclajes narcisistas infantiles, refuerza la desconexión con la alteridad, e impulsa a una verdadera propensión a la idealización de discursos capaces de relanzar la ambivalencia y la agresividad de estas posiciones subjetivas de carácter principalmente narcisista.

Siguiendo esta línea de interpretación, en el plano colectivo, nuestro examen de este episodio de violencia nos situaría frente a una lógica superyoica que se redobla a través de la incitación a un goce que discrimina y excluye al propio Sujeto discriminador. Esta figura sádica del superyó, se podría inferir, reintroduciría narraciones culturales y sociales que se expresan en la repetición del esquema dominante-dominado/abusador-abusado, subterráneo para una juventud marginalizada que debe sostener el ideal de ser parte de un modelo de sociedad que opera desde el imperativo de la segregación y la jerarquía social (Canales, 2007). Es posible concluir, en este sentido, que el asesinato del joven homosexual por jóvenes marginalizados permite observar el funcionamiento de una lógica superyoica, de índole cultural, que triunfa cuando redobla la dinámica de la segregación, y que se refuerza por la vía de la exigencia de violencia. Así discernido, el ataque y tortura del joven D. Z. sería demostrativo de un odio excesivo hacia lo que este joven vehiculiza como figura de lo desechado, como si, dentro de las características extremas de este crimen, los jóvenes atacantes hubiesen ensayado trazar los bordes imaginarios de su propia exclusión.

\section{Una hipótesis sobre la relación entre el trabajo de la transmisión psíquica y el posicionamiento subjetivo frente al superyó cultural durante la adolescencia}

Es posible indicar que el desarrollo de los procesos de historización y transmisión durante la adolescencia se encuentran enlazados con la construcción de figuras de terceridad abiertas a los intercambios simbólicos, de modo que la elaboración y construcción de este espacio narrativo conllevaría, por una parte, la posibilidad de sobrepasar las características imaginarias del mundo infantil y por otra parte la posibilidad de crear nuevas figuras discursivas, mediante las cuales establecer una cierta distancia subjetiva frente a los mandatos del Otro social, propiciando de esta forma el examen y deconstrucción del superyó grupal.

Nos permitimos en este punto utilizar la noción de superyó grupal, con el objeto de remarcar la interrelación entre los procesos de segregación y marginalización social presentes en la sociedad chilena contemporánea y la conformación de grupos cerrados o sectas que se organizan bajo la forma de una aceptación acrítica de normas particulares. En este punto, en concordancia con la perspectiva de análisis desarrollada por L. Sciara (2011), consideramos posible reconocer en estas formas de vinculación grupal, la proliferación de mecanismos de identificación imaginaria que se establecen en oposición tanto la unidad de la Ley social como a las limitaciones inherentes a la condición simbólica del hombre. En esta misma perspectiva, como lo indica Dany-Robert Dufour (2003), nos es posible reconocer, en formaciones imaginarias como la banda, formas de sometimiento del individuo a ideales y trazas identitarias capaces de borrar las particularidades individuales. En consecuencia, la noción de superyó grupal, la utilizamos con el objeto de reconocer la emergencia de disposiciones superyoicas que, sin adquirir el carácter de un gran relato capaz de interpelar al conjunto de la sociedad, promueve formas ambivalentes y agresivas de vinculación a la alteridad entre los individuos pertenecientes al grupo. Así, consideramos que la alienación a micro relatos grupales sería una de las formas de expresión, de las destinaciones imaginarias que delimitan los procesos de subjetivación de adolescentes y jóvenes contemporáneos.

En este sentido, se debe admitir que la posibilidad de diferenciar las propias actuaciones, respecto de aquellas propiciadas por los mandatos del grupo, requiere también la apertura de un trabajo de singularización de las posiciones ético-morales de los adolescentes, guiada por los esfuerzos de apropiación de la historia personal, familiar y social. Partiendo de esta lectura, la incapacidad de oponerse a los imperativos e ideales del grupo de referencia sería concordante con la detención del proceso de historización presente en la dialéctica de la persona, de suerte que esta incapacidad sería demostrativa de la adopción de una suerte de lengua del grupo, que se expresaría a través de la repetición de actos de violencia. Así, la imposibilidad de dar curso a un trabajo de habilitación moral capaz de singularizar las posiciones de estos jóvenes frente al superyó grupal sería asimismo demostrativa de sus graves dificultades para posicionarse, en tanto que individuos y frente a otros, en espacios narrativos de elaboración, a la vez intersubjetivos e intergeneracionales.

Por consiguiente, se trata de subrayar la importancia clínica de la cuestión de la legitimación de las normas, ideales o imperativos sociales y grupales, en tanto que parte del proceso de construcción de una discursividad personal que posicione al adolescente frente al Otro social. De esta manera, el ejercicio de cuestionamiento de las normas y de los imperativos del superyó grupal permitiría crear una ruptura entre las posiciones del sujeto y los imaginarios sociales, que por una parte permitiría consolidar las dinámicas de transmisión psíquica, y por otra parte permitiría constituir nuevos emplazamientos subjetivos, representativos de movimientos de subjetivación abiertos a la alteridad. En esta perspectiva, los procesos de historización de la adolescencia dependerían, por un lado, de la posibilidad de distanciamiento frente a las exigencias e ideales del superyó grupal, y, por otro lado, de la puesta en marcha de procesos sublimatorios, tendientes a reconocer la incompletud del Otro y la negatividad inherente a los procesos de simbolización. 


\section{A modo de conclusión}

El trabajo de la adolescencia articula lo individual y lo colectivo, y permite reconocer la conformación de un espacio de simbolización y de mediación entre la singularidad de la experiencia subjetiva y el orden colectivo. Así, durante la adolescencia lo individual y lo colectivo se religan, revelando la importancia de conjugar el trabajo de estructuración psíquica del sujeto, con el tratamiento social e intersubjetivo de las referencias imaginarias de la sociedad.

En esta perspectiva, sería posible indicar que la violencia y la agresividad del grupo de jóvenes que asesinaron a Daniel Zamudio puede ser explicada como una manifestación, que colindante entre lo psicológico y lo social, permite apreciar las dificultades para constituir una identidad fuera de un ambiente social atravesado por narraciones que legitiman la violencia como modo de acción y de expresión. Estas dificultades, se podría inferir, expresarían el impedimento de analizar el orden social instituyente, de un modo que permita salir de una simple reproducción de las coordenadas, subjetivas y sociales, dentro de las cuales emergen como sujetos juveniles.

Concordante con esta perspectiva, la propuesta desarrollada ha buscado contribuir a la conformación de un esquema de interpretación capaz de interrogar las formas de anudamiento entre la estructura que comanda las simbolizaciones del sujeto y las mediaciones individuales y socio simbólicas, en donde el adolescente conquista su particularidad frente a los requerimientos colectivos. De este modo, el crimen del joven homosexual, que ha permitido apuntalar nuestras reflexiones, podría eventualmente establecer ciertos ejes de reflexión para otros casos de violencia juvenil, en donde se pueda vislumbrar la intervención de factores sociales, como la pobreza o la segregación, y aspectos familiares e individuales, como la precariedad del posicionamiento simbólico.

En este sentido, se podría reafirmar, para concluir, la importancia de diseñar modelos de lectura que no reduzcan el análisis de las expresiones de violencia juvenil a un nivel de interpretación solamente sociohistórico o psicológico. A este respecto, por una parte, se debe remarcar el carácter simbolizante de los procesos de historización abiertos en la adolescencia y su importancia para la apropiación y transmisión de las discursividades presentes en la sociedad. Por otra parte, se debe recalcar la importancia de comprender la noción de superyó grupal desde un enfoque que no la reduzca a una consciencia moral, en cuanto moviliza también la pregnancia y el empuje de los ideales que fuerzan al sujeto a identificaciones imaginarias, que obturan los procesos de historización y subjetivación.

En efecto, como es posible vislumbrar mediante el estudio de este hecho de violencia, las graves dificultades de los jóvenes para posicionarse y cuestionar la legitimidad de los requerimientos del superyó grupal, las encontramos en la alianza entre el goce que es recuperado por el superyó individual y los ideales segregativos y jerárquicos de la sociedad chilena. Así discernidas, estas manifestaciones de violencia juvenil no se apuntalan solamente en las contradicciones del orden histórico y social - en este caso en las contradicciones del proceso de modernización post dictadura de la sociedad chilenasino que, principalmente, se alimentan de un ánimo vergonzante de existir (Douville, 2013, 2015) que nos anoticia de la precariedad de la inscripción de muchos jóvenes y adolescentes contemporáneos en el orden de intercambios simbólicos e intersubjetivos presentes en las sociedades contemporáneas.

Se trata pues, de inspeccionar frente a estas problemáticas la interrelación de múltiples factores que, en su conjunto, hacen posible, sino comprender, al menos vislumbrar la raíz profunda de estas violencias juveniles. Violencias demostrativas de un fuerte impulso al obnubilamiento por ideales de la destrucción y de la nada, y que, a contrasentido de los procesos de elaboración abiertos por la adolescencia, se difuminan en representaciones imaginarias de todo poder y de todo goce, impidiendo la apertura a la alteridad y al fundamento simbólico de las relaciones humanas.

\title{
Idealization and psychic transmission in adolescence: interpretation of an event of violence in Chile
}

\begin{abstract}
The purpose of this article is to examine the murder of a young homosexual committed in Chile in 2012 and analyze this event of violence in relation to the imaginaries of contemporary Chilean society and to the processes of idealization and psychic transmission. With this objective we develop a theoretical interpretation that associates psychoanalytic elaborations on the role of the cultural superego and of idealization during the adolescent passage and the interrelation between the ethnical-political dialectic and the ethical-moral dialectic in the Theory of Mediation. The proposed association highlights the power of the social ideals of control and rejection of the other on the processes of subjective appropriation of adolescence. Thus, we propose an approach to this event of violence that seeks to interrelate the psychic and interpsychic processes of adolescence and the subjectivation processes that constitute the social experience, of the adolescents and young people of today's Chile.
\end{abstract}

Keywords: adolescent passage, person, violence, idealization and transmission. 


\section{Idéalisation et transmission psychique à l'adolescence : Interprétation d'un événement de violence au Chili}

Résumé: L'objectif de cet article est d'examiner le meurtre d'un jeune homosexuel perpétré au Chili en 2012 et d'articuler cet événement de violence aux imaginaires de la société chilienne contemporaine et aux processus d'idéalisation et de transmission psychique. Dans ce but, une interprétation théorique est développée qui relie les élaborations psychanalytiques sur le rôle du surmoi culturel et de l'idéalisation au cours du passage adolescent et le croisement de la dialectique ethno-politique et de la dialectique éthique-morale dans la Théorie de la Médiation. Le lien proposé met en évidence le pouvoir des idéaux sociaux de domination et de rejet de l'autre sur les processus d'appropriation subjective de l'adolescence. Donc, il est proposé une approche de cet événement de violence qui cherche à entrelacer les processus psychiques et interpsychiques de l'adolescence et les processus de subjectivation qui constituent l'expérience sociale des adolescents et des jeunes au Chili actuel.

Mots clés: passage adolescent, personne, violence, idéalisation et transmission.

\section{Idealização e transmissão psíquica na adolescência: interpretação de um caso de violência no Chile}

Resumo: Este artigo se propõe a analisar o homicídio de um jovem homossexual, fato ocorrido no Chile em 2012, e articular este evento de violência com certos imaginários da sociedade chilena contemporânea, bem como aos processos de idealização e transmissão psíquica. A partir desse objetivo, desenvolver-se-á uma interpretação teórica vinculada a elaborações psicanalíticas sobre o papel do superego cultural e da idealização durante a fase adolescente, fazendo também um intercruzamento entre a dialética étnico-política e a dialética ético-moral, na Teoria da Mediação. A referida perspectiva destaca uma maior incidência dos ideais sociais de controle e exclusão do outro sobre os processos de apropriação subjetiva da adolescência. Dessa forma, intenta-se uma aproximação a este caso violento, buscando tecer um emaranhado de processos psíquicos e interpsíquicos da adolescência e os processos de subjetivação constitutivos da experiência social de adolescentes e jovens no Chile de agora.

Palavras-chave: passagem adolescente, pessoa, violência, idealização e transmissão.

\section{Referencias}

Aceituno, R., Miranda, G., \& Jiménez, Á. (2012). Experiencias del desasosiego: salud mental y malestar en Chile. Revista Anales de la Universidad de Chile, (3), 87-102.

Aravena Reyes, A., \& Baeza, M. A. (2013). Violencia simbólica en el Chile contemporáneo: estrategias de respuesta en relaciones de alteridad. Revista Internacional de Sociología, 71(3), 543-565.

Brackelaire, J. L. (2009). La personne en suspens : singularisme moderne et courts-circuits dans la transmission. In M. Gauchet, \& J. C. Quentel, Histoire du sujet et théorie de la personne (pp. 123-144). Rennes, France: Presses Universitaires de Rennes.

Canales, M. (2007). Ni pobres ni incluidos: ¿nueva cuestión social? Revista de Sociología, (21), 193-207.

Cuarto Tribunal de Juicio Oral en lo Penal de Santiago (2013). Individualización de audiencia de lectura de sentencia. Recuperado de https://goo.gl/WQ4pfa

Douville, O. (2001). Pour introduire l'idée d'une mélancolisation du lien social. Cliniques Méditerranéennes, (1), 239-262.

Douville, O. (2005). Aujourd'hui le structuralisme? Figures de la Psychanalyse, (2), 11-26.

Douville, O. (2013). Situations et destinées des enfants et des adolescents dans la guerre en Afrique. Études Sur la Mort, (2), 55-68.
Douville, O. (2015). De la honte à l'indifférence : situations d'enfants et d'adolescents. . . sous la guerre, en Afrique. La Clinique Lacanienne, (1), 199-212.

Duarte, D. (2007). Suicidio en Chile: un signo de exclusión. Santiago, Chile: Universitaria.

Dufour, D. R. (2003). L'art de réduire les têtes : sur la nouvelle servitude de l'homme libéré, à l'ère du capitalisme total. Paris, France: Denoël.

Fluxá, R. (2014). Solos en la noche: Zamudio y sus asesinos. Santiago, Chile: Catalonia.

Freud, S. (1978). Psicología de las masas y análisis del yo. In J. Strachey (Ed.), Obras completas: Sigmund Freud (J. L. Etcheverry, trad., Vol. 18, pp. 63-136). Buenos Aires, Argentina: Amorrortu. (Trabalho original publicado em 1921)

Freud, S. (1978). El malestar en la cultura. In J. Strachey (Ed.), Obras completas: Sigmund Freud (J. L. Etcheverry, trad., Vol. 21, pp. 22-116). Buenos Aires, Argentina: Amorrortu. (Trabalho original publicado em 1929)

Gagnepain, J. (1993). Huit leçons d'introduction a la theorie de la mediation. Montpeyroux: Institut Jean Gagnepain. Recuperado de https://goo.gl/daeT4D

Gutton, P. (2002). Violence et adolescence. Paris, France: Press Éditions.

León, F. (2015). Estándares conceptuales y cargas procesales en el litigio antidiscriminación: análisis crítico de la 
jurisprudencia sobre Ley Zamudio entre 2012 y 2015. Revista de Derecho, (28), 145-167.

Lesourd, S. (2004). La déconstruction-reconstruction des systèmes référentiels. In P. Gutton, \& S. Bourcet, $L a$ naissance pubertaire : l'archaïque et son devenir (pp. 99-126). Paris, France: Dunod.

Lesourd, S. (2006). Comment taire le sujet? Des discours aux parlottes libérales. Ramonville Saint-Agne, France: Érès.

Mijolla-Mellor, S. (2012). Traité de la sublimation. Paris, France: Presses Universitaires de France.

Mayol, A., Azócar, C., \& Azócar, C. (2012). El Chile profundo: cultura de la desigualdad en Chile contemporáneo. Santiago, Chile: Universitaria.

Moulian, T. (2002). Chile actual: anatomía de un mito. Santiago, Chile: Lom.

Movilh (2014). Daniel Zamudio, hiciste historia. Recuperado de https://goo.gl/iwmiTQ

Quentel, J. C. (2011). L'adolescence aux marges du social. Bruxelles, Belgium: Fabert.
Quentel, J.C. (2015). Du social et de l'éthique, de la politique et de la morale au regard de la théorie de la médiation. Tétralogiques, (20), 21-50.

Rassial, J. J. (1996). Le passage adolescent: de la famille au lien social. Toulouse, France: Érès.

Rassial, J. J. (1999). Le sujet en état limite. Paris, France: Denoël.

Salazar, G. (1999). Raíces históricas de la violencia en Chile. Revista de Psicología, 8(2), 19.

Salazar, M., \& Valderrama, M. (2000). Dialectos en transición: política y subjetividad en el Chile actual. Santiago, Chile: Lom.

Sciara, L. (2011). Banlieues: pointe avancée de la clinique contemporaine. Toulouse, France: Érès.

Recibido: $14 / 05 / 2017$

Revisado: 06/12/2017

Aprobado: 16/03/2018 